Somayya Noori, Christie P. M. Verkleij, Marina Zajec, Pieter Langerhorst, Patricia W. C. Bosman, Yolanda B. de Rijke, Sonja Zweegman, Martijn VanDuijn, Theo Luider, Niels W. C. J. van de Donk and Joannes F. M. Jacobs*

\title{
Monitoring the M-protein of multiple myeloma patients treated with a combination of monoclonal antibodies: the laboratory solution to eliminate interference
}

https://doi.org/10.1515/cclm-2021-0399

Received April 1, 2021; accepted August 8, 2021;

published online August 16, 2021

\section{Abstract}

Objectives: The therapeutic monoclonal antibody (t-mAb) daratumumab, used to treat multiple myeloma (MM) patients, interferes with routine, electrophoretic based M-protein diagnostics. Electrophoretic response assessment becomes increasingly difficult when multiple t-mAbs are combined for use in a single patient. This is the first study to address the analytical challenges of M-protein monitoring when multiple t-mAbs are combined.

Methods: In this proof-of-principle study we evaluate two different methods to monitor M-protein responses in three

Somayya Noori and Christie P. M. Verkleij share first authorship.

Niels W. C. J. van de Donk and Joannes F. M. Jacobs share last authorship.

*Corresponding author: Joannes F. M. Jacobs, PhD, MD, Department of Laboratory Medicine, Laboratory Medical Immunology (route 469), Radboud University Medical Center, Geert Grooteplein 10, 6525 GA Nijmegen, The Netherlands, Phone: +31 (0)24 3614625, Fax: +31 (0)24 3619415, E-mail: H.Jacobs@Radboudumc.nl

Somayya Noori, Martijn VanDuijn and Theo Luider, Department of Neurology, Erasmus University Medical Center, Rotterdam, The Netherlands

Christie P. M. Verkleij, Patricia W. C. Bosman, Sonja Zweegman and Niels W. C. J. van de Donk, Department of Hematology, Amsterdam University Medical Center, Vrije Universiteit Amsterdam, Amsterdam, The Netherlands

Marina Zajec, Department of Neurology, Erasmus University Medical Center, Rotterdam, The Netherlands; and Department of Clinical Chemistry, Erasmus University Medical Center, Rotterdam, The Netherlands. https://orcid.org/0000-0001-9874-2771

Pieter Langerhorst, Department of Laboratory Medicine, Radboud University Medical Center, Nijmegen, The Netherlands Yolanda B. de Rijke, Department of Clinical Chemistry, Erasmus University Medical Center, Rotterdam, The Netherlands
MM patients, who receive both daratumumab and nivolumab. The double hydrashift assay aims to resolve t-mAb interference on immunofixation. The MS-MRD (mass spectrometry minimal residual disease) assay measures clonotypic peptides to quantitate both M-protein and $\mathrm{t}-\mathrm{mAb}$ concentrations.

Results: After exposure to daratumumab and nivolumab, both t-mAbs become visible on immunofixation electrophoresis (IFE) as two IgG-kappa bands that migrate close to each other at the cathodal end of the $\gamma$-region. In case the $\mathrm{M}$-protein co-migrates with these $\mathrm{t}$-mAbs, the observed interference was completely abolished with the double IFE hydrashift assay. In all three patients the MS-MRD assay was also able to distinguish the M-protein from the t-mAbs. Additional advantage of the MS-MRD assay is that this multiplex assay is more sensitive and allows quantitative M-protein-, daratumumab- and nivolumab-monitoring.

Conclusions: Daratumumab and nivolumab interfere with electrophoretic M-protein diagnostics. However, the M-protein can be distinguished from both t-mAbs by use of a double hydrashift assay. The MS-MRD assay provides an alternative method that allows sensitive and simultaneous quantitative monitoring of both the M-protein and t-mAbs.

Keywords: daratumumab; hydrashift; immunofixation electrophoresis; mass spectrometry; monoclonal antibody; M-protein; multiple myeloma; nivolumab; serum protein electrophoresis; therapeutic drug monitoring.

\section{Introduction}

Therapeutic monoclonal antibodies (t-mAbs) have become increasingly important in the treatment of hematological malignancies [1], including multiple myeloma (MM) [2]. Daratumumab is a fully human anti-CD38 IgG1-kappa mAb, and is approved for the treatment of newly diagnosed [3-5] and relapsed/refractory [6, 7] (RR) MM. Daratumumab has 
direct on-tumor effects, but also immunomodulatory effects through the elimination of CD38-positive immune suppressor cells such as regulatory T-cells [8]. The fully human IgG4-kappa t-mAb nivolumab binds to the immune checkpoint programmed death (PD)-1, which is present on the cell surface of T-cells and NK-cells. Blockade of PD-1 by nivolumab improves the host-anti-tumor immune response in several types of cancer. As a single agent, nivolumab induced durable stable disease in $67 \%$ of RRMM [9], but no objective responses were observed. Preclinical studies have shown that immune modulation through dual targeting of CD38 and PD-1 resulted in enhanced anti-MM activity, compared to single targeting [10]. Several phase 2 clinical trials investigating these combinations are ongoing in RRMM (NCT03184194, NCT01592370).

Therapeutic mAbs can appear on electrophoretic scans as small monoclonal bands [11,12]. In routine diagnostics it may be challenging to distinguish the human(ized) $t-m A b$ from the endogenous M-protein due to co-migration. As a result, the IMWG response criteria have been modified to account for the presence of $\mathrm{t}-\mathrm{mAb}$ interference [13]. However, co-migration of $\mathrm{t}-\mathrm{mAb}$ and the endogenous M-protein can result in the inability to accurately assess therapeutic complete responses $[11,14]$. Complete response is used in clinical trials as a surrogate for improved outcome, since it is significantly correlated with survival after any given treatment $[15,16]$. Electrophoretic interference of $t-m A b$ can be overcome using an antibody targeting the t-mAb to alter the serum protein electrophoresis (SPEP) migration. For daratumumab, a so-called shift-assay has been realized $[17,18]$. The commercial version is named the Hydrashift assay (Sebia), which was recently FDA-approved to mitigate possible antibody interference [19]. However, this assay is specific for daratumumab and will not eliminate immunofixation electrophoresis (IFE) interference caused by other t-mAbs. Response assessment with electrophoretic M-protein diagnostics will become increasingly difficult when MM patients are treated with a combination of $\mathrm{t}$-mAbs, a strategy that is explored in multiple clinical studies.

Mass spectrometry (MS) has recently been introduced as an alternative method to identify and monitor M-proteins with increased sensitivity compared to routine M-protein diagnostics [20]. Initial publications convincingly show that MS methodology to measure the endogenous M-protein is not affected by t-mAb interference [21-23]. However, this was never shown in patients exposed to more than one t-mAb.

In this proof-of-principle study we evaluate two different methods to monitor M-protein responses in three MM patients, who receive both daratumumab and nivolumab (NCT03184194). We demonstrate that both daratumumab and nivolumab, dosed at therapeutic blood levels, can be detected as two monoclonal bands in serum IFE. The endogenous M-protein can be distinguished from these two t-mAb bands using a double hydrashift assay. In addition, we show that the MS-MRD (mass spectrometry minimal residual disease) assay provides a method that allows sensitive and simultaneous quantitative monitoring of both the M-protein and the t-mAbs.

\section{Materials and methods}

\section{Patient samples}

Peripheral blood samples were collected from relapsed/refractory MM patients participating in the Nivo-Dara study (NCT03184194). In this study, patients received daratumumab at a dose of $16 \mathrm{mg} / \mathrm{kg}$ i.v., according to the approved schedule (weekly during the first eight weeks, then every two weeks for a period of 16 weeks, and every four weeks thereafter), in combination with nivolumab (240 mg i.v. every two weeks during the first six cycles and $480 \mathrm{mg}$ i.v. every four weeks thereafter), with or without daily low-dose cyclophosphamide (50 mg p.o.). To monitor $\mathrm{t}-\mathrm{mAb}$ fluctuations, blood samples were collected directly prior to the administration of both t-mAbs (pre-infusion) and at the end of the infusion (post-infusion). Serum was separated from whole blood and stored at $-20{ }^{\circ} \mathrm{C}$ until further analysis. The study site Ethics Committee (METc VUmc, study registration number 2017.159/NL60544.029.17) approved the protocols, which were conducted according to the principles of the Declaration of Helsinki, the International Conference on Harmonization, and the Guidelines for Good Clinical Practice. All patients provided written informed consent.

\section{M-protein diagnostics}

Routine serum protein electrophoresis (SPEP) and IFE were performed on the Hydrasys 2 system using the Hydragel 4 IF kit (Sebia). The hydrashift (Sebia) procedure is similar to that of a normal IFE, except that the mouse monoclonal $\mathrm{t}-\mathrm{mAb}$ antiserum $(5 \mathrm{~g} / \mathrm{L})$ is applied to the gel with an additional applicator. All electrophoretic methods were performed according to manufacturer's protocol. All components of the hydrashift kit have a stability of at least 24 months. The anti-idiotype antibodies against daratumumab and nivolumab are chemically modified in order to achieve anodic electrophoretic mobility. The increased electrophoretic mobility directs the anti-ideotype antibodies to the $\alpha 1$-region, away from the regions where normal immunoglobulins migrate. The sensitivity and specificity of the hydrashift assay was previously shown by Caillon et al. [24]. Supplementary Figure 1 shows the performance of the nivolumab hydrashift in three sera from healthy controls spiked with nivolumab. For the double hydrashift assay that was performed in this study, an off-protocol procedure was developed in which both anti-daratumumab and anti-nivolumab antisera were simultaneously applied to the gel with the same aforementioned applicator. In addition, serum free light chain (sFLC) measurements were 
performed using FreeLite reagents (The Binding Site) on a BNII nephelometer (Siemens), according to the manufacturer's protocol.

\section{Tryptic digestion and LC-MS}

Prior to digestion, samples were reduced for $30 \mathrm{~min}$ at $60{ }^{\circ} \mathrm{C}$ in $60 \mu \mathrm{L}$ $50 \mathrm{mM}$ ammonium bicarbonate containing $15 \% \mathrm{v} / \mathrm{v}$ acetonitrile, $0.1 \%$ Rapigest (Waters, Milford, MA), $10 \mathrm{mM}$ DTT, $10 \mathrm{nM}$ of each stable isotope labelled internal peptide standards (Pepscan B.V., Lelystad, The Netherlands) and $0.12 \mu \mathrm{L}$ patient serum. After cooling, iodoacetamide was added to $15 \mathrm{mM}$ and incubated for $30 \mathrm{~min}$ at $25{ }^{\circ} \mathrm{C}$ in darkness. After addition of $400 \mathrm{ng}$ trypsin (Promega, Madison, WI) samples were incubated overnight at $37^{\circ} \mathrm{C}$. Samples were then acidified with $1.5 \mu \mathrm{L} 25 \%$ trifluoroacetic acid, incubated for $30 \mathrm{~min}$ at $37^{\circ} \mathrm{C}$ and centrifuged for $45 \mathrm{~min}$ at $20,000 \mathrm{~g}$ and $4^{\circ} \mathrm{C}$.

\section{Mass spectrometry, LC-MS measurement}

The samples were measured on a nano-LC system (Ultimate 3000, Thermo Fisher Scientific, Munich, Germany). Five microliter sample was captured on a trap column and separated on a $75 \mu \mathrm{m}$ ID $\times 250 \mathrm{~mm}$ C18 PepMap column ( $2 \mu \mathrm{m}, 100 \AA$ pore, Thermo Fisher Scientific). Peptides were eluted with a $30 \mathrm{~min}$ gradient of $4-38 \%$ solvent $B$. Solvent A consisted of $0.1 \%$ formic acid in water and solvent B of $0.08 \%$ formic acid plus $80 \%$ acetonitrile. Peptides were ionized in a nanospray ESI source on an Orbitrap Fusion (Thermo Fisher Scientific, Bremen, Germany). Each sample was measured first with a data dependent acquisition method, then measured with targeted MS on selected peptides of the M-protein and monoclonal antibodies (Table 1) with the following parameters: isolation width of $1.4 \mathrm{~m} / z$, maximum injection time of $246 \mathrm{~ms}$ and analyzer resolution of 120,000 .

\section{Mass spectrometry, $M$-protein peptide selection}

M-protein peptides have been selected based on de novo sequencing information obtained with PEAKS Studio (Bioinformatics Solutions Inc., version 6). For the de novo search in PEAKS an error tolerance of $5 \mathrm{ppm}$ was applied, the search was performed on specific trypsin cleavages with a maximum of one missed cleavage. A database search was performed against a database of human germline immunoglobulin sequences (IMGT) [25]. With this search, unique clonal peptides are identified with amino acid alterations that are different compared to the immunoglobulin germline. Peptide quantity was assessed by labelfree analysis in Progenesis QI (version 2.0, Waters, Milford, MA). For the selection of M-protein peptides a number of criteria were evaluated: homology to the germline sequence, but at least one amino acid mutation compared to the germline sequence [26]. This is further verified with IgBlast [27]. The isobaric amino acids leucine and isoleucine cannot be distinguished in the dataset and were therefore not scored as mutations.

\section{Mass spectrometry, protein quantification}

The targeted MS data is imported into Skyline-daily (MacCoss Lab Software, version 20.1.1.175), that visualizes the identified peptide fragments and their contribution to the peptide peak signal. Supplementary Figure 2 shows an example of the chromatograms of one identified clonotypic peptide. Two control sera from a healthy serum were spiked with $5 \mathrm{~g} / \mathrm{L}$ of intact $\mathrm{t}-\mathrm{mAb}$ daratumumab or nivolumab. These sera with known concentration of t-mAb were used as calibrators for the quantification of daratumumab and nivolumab in all three patients. The concentration of daratumumab and nivolumab is calculated in every sample based on the ratio between the area of the endogenous peptide and of the stable isotope labelled (SIL) peptide peak, calculated by Skyline. The M-protein concentration is calculated based on the known M-protein concentration in the baseline sample and the ratio between peptide areas of the baseline sample and the unknown sample of the patient.

\section{Results}

\section{Both daratumumab and nivolumab interfere with routine $M$-protein diagnostics}

To investigate whether the human IgG-kappa nivolumab at therapeutic dosing interferes with M-protein diagnostics using electrophoretic methods, we selected three patients from the Nivo-Dara study, who were treated with the combination of daratumumab and nivolumab. Patient characteristics are shown in Supplementary Table 1. The left panels of Figure 1 show the IFE's at study entry (i.e. prior to t-mAb

Table 1: Selected mAb-specific peptides.

\begin{tabular}{lllrrr}
\hline Protein & Peptide sequence & Ig chain & $\mathbf{m} / \mathbf{z}$ & $\boldsymbol{z}$ & HCD collision energy, \% \\
\hline Daratumumab & GLEWVSAISGSGGGTYYADSVK & IgG-HC & 735.3551 & 3 & 30 \\
Nivolumab & ASGITFNSGMHWVR & IgG-HC & 825.3963 & 2 & 28 \\
IgG-kappa & APNLLLYASNLEAGVPSR & IgG-HC & $1,000.5260$ & 2 & 28 \\
M-protein pt 1 & EWVAVAVLYYDGSNEHYVESVK & Kappa-LC & 853.0797 & 3 & 28 \\
IgG-kappa & AAPPGQAPSLLLGASTR & IgG-HC & 885.4809 & 2 & 28 \\
M-protein pt 2 & WELGLTFSYDGDTDHYADSVK & Kappa-LC & 836.0397 & 3 & 28 \\
FLC-kappa & ASQSVGTNLAWYLHKPGQAPR & Kappa-LC & 761.0661 & 3 & 28 \\
M-protein pt 3 & LDVMTQSPATLSVSPADR & Kappa-LC & 944.4777 & 2 & 28 \\
\hline
\end{tabular}

Mutations in the M-protein peptides compared to the germline sequence are underlined. HCD, higher-energy collisional dissociation; HC, heavy chain; FLC, free light chain; $m / z$, mass to charge ratio. 
administration) for patients 2 and 3. For patient 1 the entry sample was not available and the earliest treatment sample (cycle 2) is shown. Patients 1 and 2 had an IgG-kappa $\mathrm{M}$-protein migrating at the anodal end of the $y$-region and patient 3 had an FLC-kappa M-protein migrating at the cathodal end of the $y$-region. After exposure to daratumumab and nivolumab (see Table 2 for therapeutic details), two new IgG-kappa bands appear close to each other at the cathodal end of the $y$-region in all three patients (Figure 1, right panels). Furthermore, in all three patients we observe therapeutic responses (Table 2).
With two t-mAbs migrating close to each other, the chance increases that the endogenous M-protein co-migrates with the t-mAbs. This hampers accurate quantification of the M-protein using SPEP. Additionally, if a patient with an IgG-kappa or FLC-kappa M-protein responds well to therapy, it is no longer possible to make valid clinical response calls that meet the IMWG criteria for (stringent) complete response ((s)CR). The latter is the case in patient 3 since the FLC-kappa M-protein comigrates with daratumumab and nivolumab. After treatment, it is not possible to properly interpret whether this
A

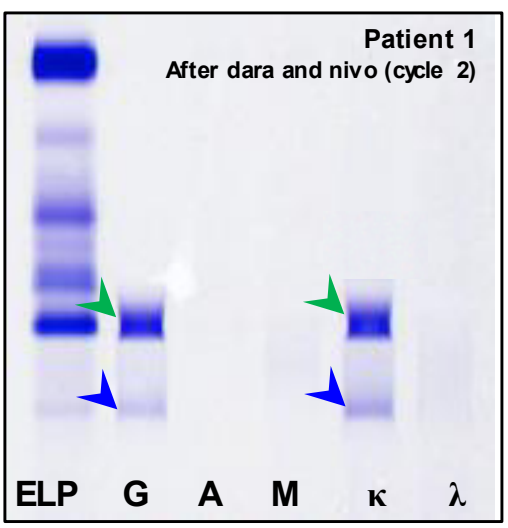

B

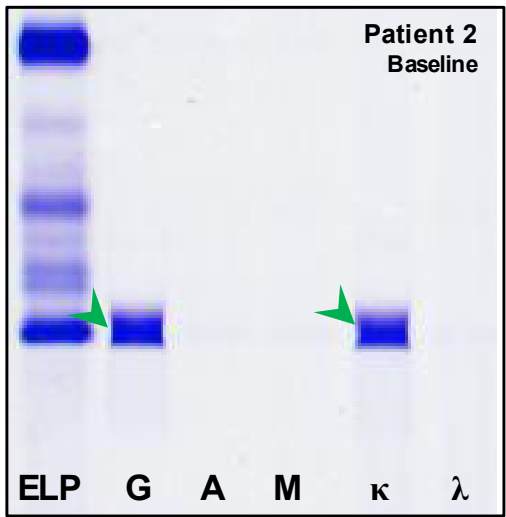

C

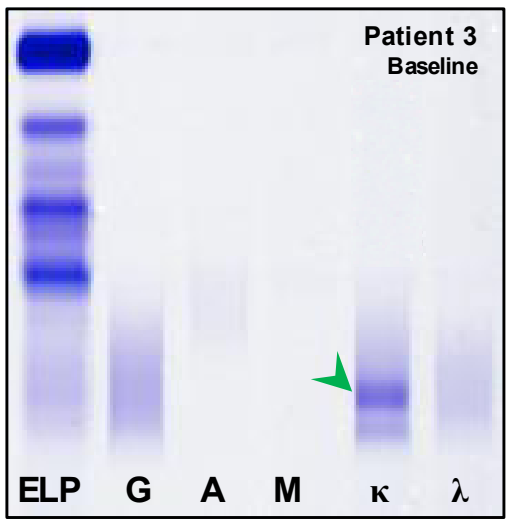

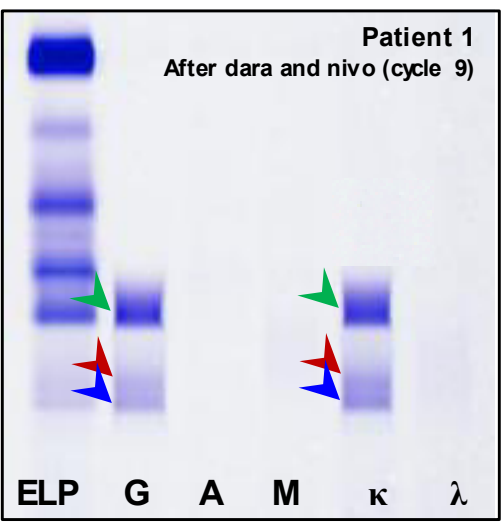
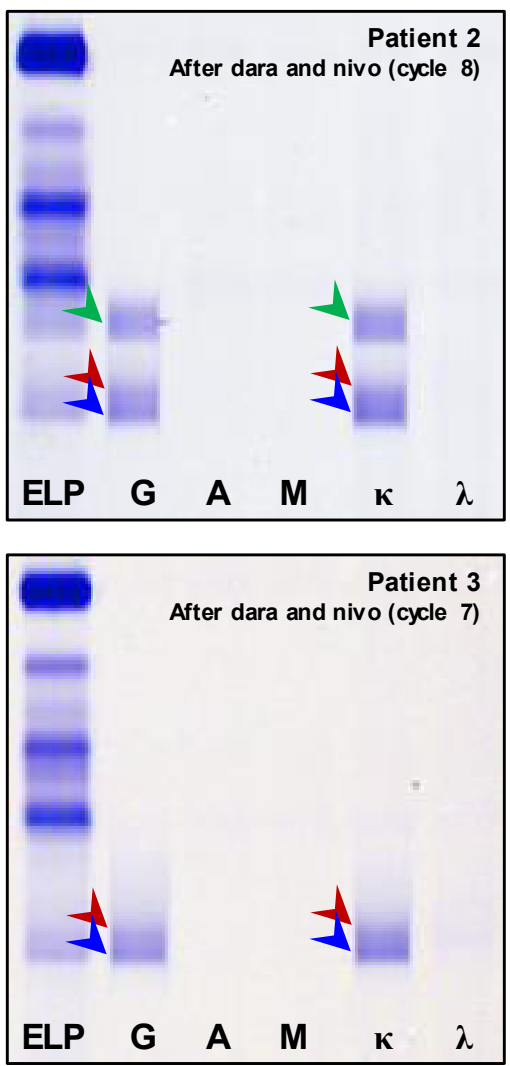

Figure 1: Detection of daratumumab and nivolumab by serum immunofixation electrophoresis.

Patients 1 (A) and 2 (B) both have an IgG-kappa M-protein (left panels). After exposure to daratumumab and nivolumab the endogenous $M$-protein band becomes less pronounced and two new IgG-kappa bands appear close to each other at the cathodal end of the $\gamma$-region. (C) At baseline an FLC-kappa M-protein is visible in patient 3 (left panel). Since the M-protein comigrates with daratumumab and nivolumab, it is no longer possible to distinguish the M-protein from the two t-mAbs. The M-protein, daratumumab and nivolumab are highlighted in each panel with respectively green, blue and red arrowheads. 
Table 2: Patient-specific therapeutic details and overview of MS results.

\begin{tabular}{|c|c|c|c|c|c|c|c|c|c|}
\hline \multirow[t]{2}{*}{ Pt } & \multirow{2}{*}{$\begin{array}{l}\text { Therapy } \\
\text { cyclus }\end{array}$} & \multirow{2}{*}{$\begin{array}{l}\text { Administered dose } \\
\text { of Dara }\end{array}$} & \multirow{2}{*}{$\begin{array}{l}\text { Administered dose } \\
\text { of Nivo }\end{array}$} & \multirow{2}{*}{$\begin{array}{l}\text { M-protein } \\
\text { type }\end{array}$} & \multirow{2}{*}{$\begin{array}{l}\text { M-protein } \\
\text { concentration }\end{array}$} & \multirow{2}{*}{$\begin{array}{l}\text { Timing blood } \\
\text { sampling }^{\mathrm{a}}\end{array}$} & \multicolumn{3}{|c|}{ MS results, $g / L$} \\
\hline & & & & & & & M-protein & Dara & Nivo \\
\hline \multirow[t]{3}{*}{1} & C2D1 & $1,080 \mathrm{mg}$ & $240 \mathrm{mg}$ & IgG-K & $7.1 \mathrm{~g} / \mathrm{L}$ & Pre $^{b}$ & 7.1 & 0.43 & 0.13 \\
\hline & C9D1 & $1,080 \mathrm{mg}$ & $480 \mathrm{mg}$ & $\lg G-K$ & $2.4 \mathrm{~g} / \mathrm{L}$ & Pre & 2.2 & 0.12 & 0.26 \\
\hline & & & & & & Post $^{\mathrm{b}, \mathrm{c}}$ & & 0.48 & 0.50 \\
\hline \multirow[t]{3}{*}{2} & Baseline & - & - & IgG-K & $14.5 \mathrm{~g} / \mathrm{L}$ & $\operatorname{Pre}^{b}$ & 14.5 & n.d. & n.d. \\
\hline & C8D1 & $1,240 \mathrm{mg}$ & $480 \mathrm{mg}$ & IgG-K & $<1 \mathrm{~g} / \mathrm{L}$ & Pre & 1.6 & 0.61 & 0.28 \\
\hline & & & & & & Post ${ }^{b, c}$ & & 1.19 & 0.63 \\
\hline \multirow[t]{5}{*}{3} & Baseline & - & - & $\mathrm{FLC}-\mathrm{K}$ & $2,450 \mathrm{mg} / \mathrm{L}^{\mathrm{d}}$ & Pre $^{\mathrm{b}}$ & 2.45 & n.d. & n.d. \\
\hline & C6D1 & $1,360 \mathrm{mg}$ & $240 \mathrm{mg}$ & FLC-K & $69 \mathrm{mg} / \mathrm{L}^{\mathrm{d}}$ & Pre & 0.075 & 0.39 & 0.20 \\
\hline & & & & & & Post & 0.075 & 0.93 & 0.37 \\
\hline & C7D1 & $1,360 \mathrm{mg}$ & $480 \mathrm{mg}$ & $\mathrm{FLC}-\mathrm{K}$ & $46 \mathrm{mg} / \mathrm{L}^{\mathrm{d}}$ & Pre & 0.050 & 0.46 & 0.18 \\
\hline & & & & & & Post ${ }^{b, c}$ & 0.055 & 0.86 & 0.40 \\
\hline
\end{tabular}

${ }^{\mathrm{a} B e f o r e}$ (pre) or directly after (post) administration of both t-mAbs. ${ }^{\mathrm{b}}$ IFE results are shown in Figure 1. ${ }^{\mathrm{C}}$ IFE results are shown in Figure 3.

${ }^{\mathrm{d}}$ Measured using FLC-assay. Pt, patient; Dara, daratumumab; Nivo, nivolumab; C, cyclus; D, day; k, kappa; n.d., not detected; MS, mass spectrometry.

patient is IFE negative or positive because of the t-mAb interference.

\section{Double daratumumab/nivolumab hydrashift assay resolves interference}

A daratumumab-specific hydrashift assay was recently developed to abrogate daratumumab interference (Figure 2). The double hydrashift assay is similar to a normal IFE, except that antisera against daratumumab and nivolumab are applied to the gel using an additional applicator. The antisera bind to the $\mathrm{t}-\mathrm{mAb}$ and shift both biologics towards the $\alpha$-region, where they no longer interfere with the interpretation of the M-protein. A remaining band in the region of interest indicates that the IgG-kappa M-protein is still present (Figure 2D, representation of a positive double hydrashift). A negative result in a double hydrashift assay shows the complete disappearance of the M-protein from the $y$-region (Figure 2E).

Proof-of-principle of the double daratumumab/nivolumab hydrashift assay is shown for all three patients in Figure 3. When a daratumumab hydrashift assay is performed in patients 1 and 2 (Figure 3A, B, middle panels), we observe that daratumumab-antisera complexes are shifted and two sharp bands, that represent the M-protein and nivolumab, remain visible. With the double daratumumab/nivolumab hydrashift assay (Figure 3A, B, right panels), only the $M$-protein band remains visible in the $y$-region in serum samples from patients 1 and 2. Based on the comparison of the baseline data (Figure 1 left panels) and assessment of therapy response using the double hydrashift assay (Figure 3 right panels) it was concluded that patient 1 achieved a partial response and patient 2 a very good partial response. The added value of the double daratumumab/nivolumab hydrashift assay is most pronounced for patient 3 , because of comigration of the M-protein and the t-mAbs (Figure 3B). With a daratumumab hydrashift assay (middle panel), IFE interpretation of the FLC-kappa is still masked by the comigrating nivolumab band. With the double daratumumab/nivolumab hydrashift (right panel) both interfering t-mAbs are shifted away from the $y$-region and it is clear that the FLC-kappa M-protein is no longer visible in the IFE. These results, combined with a negative urine IFE and $<5 \% \mathrm{MM}$ cells in the bone marrow, indicate that this patient had achieved a CR.

\section{MS-MRD assay can simultaneously quantify t-mAbs and M-protein concentrations}

We further investigated whether our previously published MS-MRD assay in which we monitor clonotypic peptides of the M-protein [21, 28], could be expanded with simultaneous quantification of multiple $t-m A b$. Table 1 provides an overview of the clonotypic peptides and MS-characteristics of both the t-mAbs and the unique M-proteins from the three different patients. In all three patients the M-protein concentrations could be monitored independently of the t-mAb titers (Table 2). The M-protein and both biologics are all measured in one multiplex MS-assay. Additional advantage of the MS-MRD assay is the increased sensitivity 
A

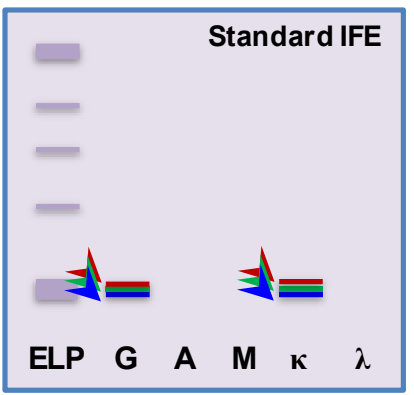

B

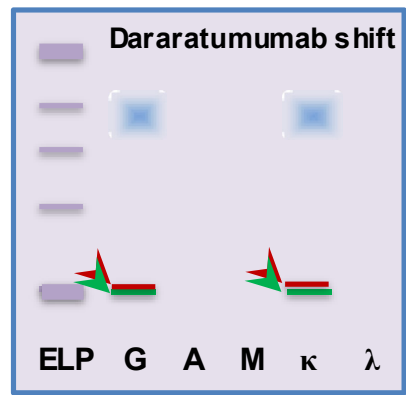

C

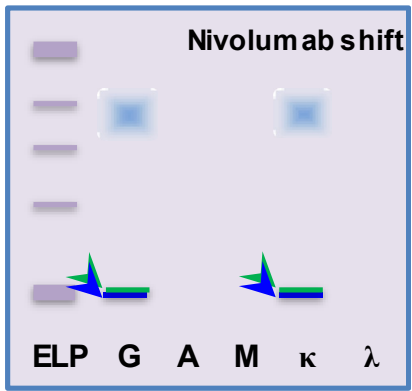

D

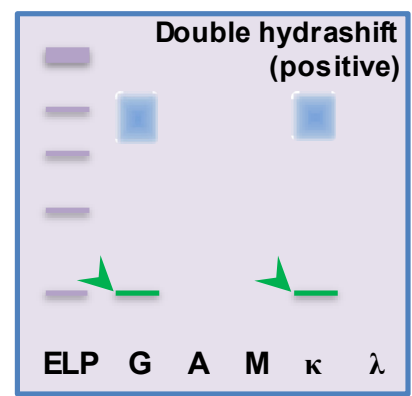

$E$

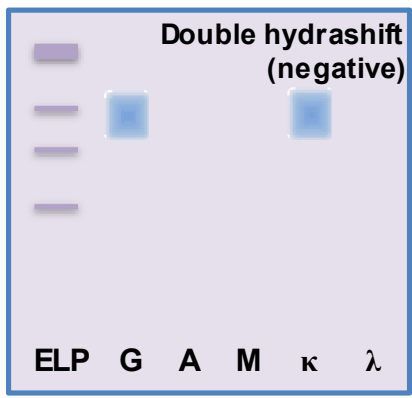

$\mathrm{F}$

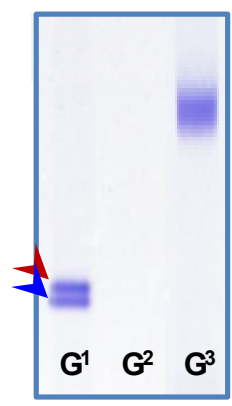

Figure 2: Schematic presentation of double IFE Hydrashift Assay.

(A) The endogenous IgG-kappa M-protein (green clonal band) comigrates with the t-mAb's daratumumab (blue) and nivolumab (red). Interpretation is impossible as all three IgG-kappa monoclonal antibodies accumulate into one broad band. (B) Single daratumumab hydrashift assay, only daratumumab is shifted towards the $\alpha$-region. Nivolumab band still interferes with $M$-protein. (C) Single nivolumab hydrashift assay, only nivolumab is shifted towards the $\alpha$-region. Daratumumab band still interferes with $M$-protein. (D) The double hydrashift assay causes both the t-mAb bands to shift towards the $\alpha$-region. A remaining band in the region of interest indicates that the $M$-protein (green) is still present. (E) A hydrashift negative result shows the complete disappearance of the monoclonal IgG-kappa bands from the $\gamma$-region and indicates that the band visible on standard IFE was due entirely to t-mAbs. (F) anti-IgG IFE of daratumumab and nivolumab spiked in saline both at $1 \mathrm{~g} / \mathrm{L}$ (lane $\mathrm{G}^{1}$ ); shift reagents only at regular concentration of $5 \mathrm{~g} / \mathrm{L}$, this provides no IFE signal since the IFE anti-sera detect only human IgG $\left(\mathrm{G}^{2}\right)$; daratumumab and nivolumab spiked in saline both at $1 \mathrm{~g} / \mathrm{L}$ in the presence of shift reagents $\left(\mathrm{G}^{3}\right)$.

for M-protein quantification. The lower limit of electrophoretic M-protein quantification is $\sim 2 \mathrm{~g} / \mathrm{L}[29,30]$, in Table 2 it is shown that MS-MRD can monitor mAb concentrations down to $0.12 \mathrm{~g} / \mathrm{L}$. This allowed quantitative monitoring of both the M-protein and the t-mAb well beyond the limit of quantification of conventional M-protein diagnostics. The t-mAb MS-titers were always higher in samples taken directly after t-mAb infusion as compared to the MS-titers measured prior to t-mAb infusion (Table 2). The M-protein concentration quantified using MS-MRD closely resembled the M-protein concentration measured with routine $\mathrm{M}$-protein diagnostics. In the two baseline samples, we did not detect any t-mAb MS-signal which is in accordance with the fact that these patients were not yet exposed to daratumumab and nivolumab.

\section{Discussion}

We demonstrate that daratumumab and nivolumab, dosed at therapeutic blood levels, can both be detected by using serum IFE. Both t-mAbs migrate close to each other to form a broad band at the cathodal end of the $y$-region. The combined use of $t-m A b s$ further increases the chance for interference with routine M-protein follow-up that is part of the IMWG criteria to assess treatment response in patients with MM [31, 32]. Incorrect response assessment due to interference in SPEP/IFE assays, most often a misclassification between very good partial response (VGPR) and complete response (CR), may lead to underestimation of prognosis and possible overtreatment of individual patients. 
A
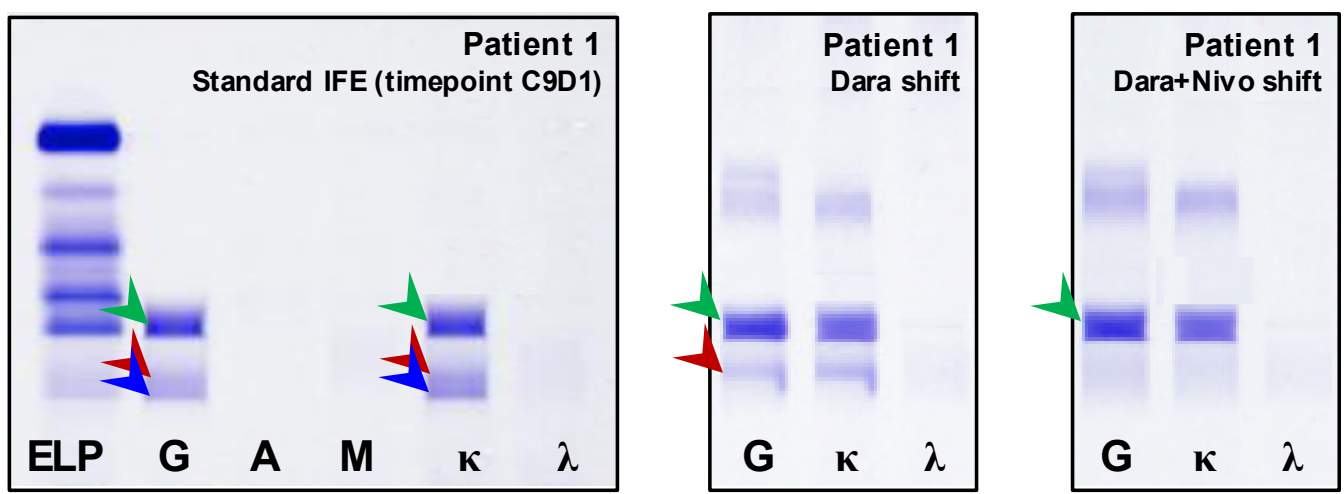

B
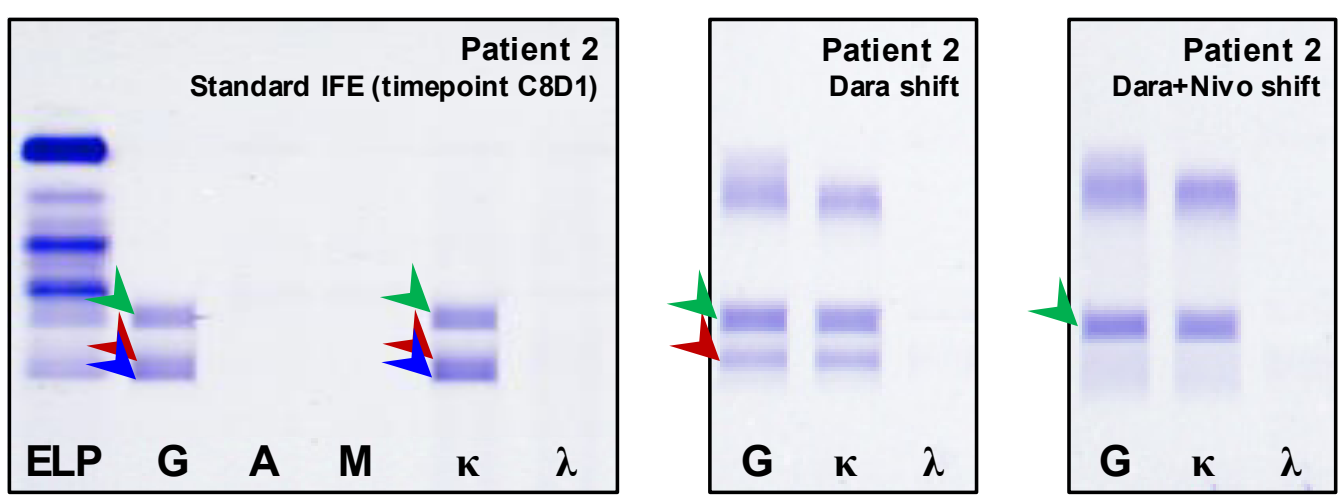

C
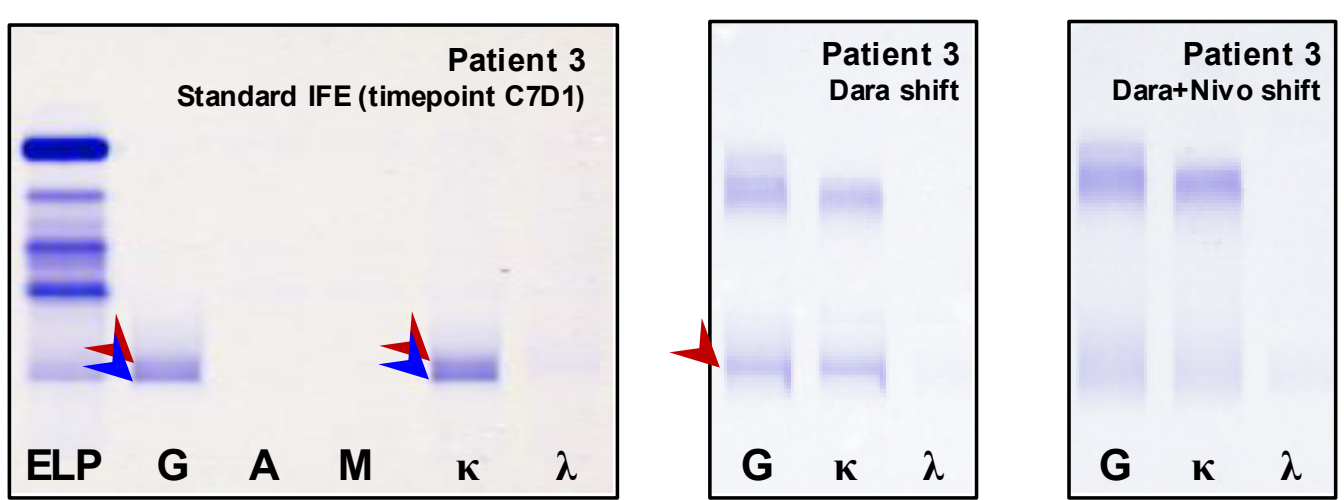

Figure 3: Double IFE Hydrashift Assay to assess therapy response.

During treatment of patients 1 (A) and 2 (B) with daratumumab and nivolumab, the endogenous IgG-kappa M-protein is clearly visible as monoclonal band (left panels). During hydrashift of daratumumab (middle) and daratumumab plus nivolumab (right) the endogenous M-proteins do not shift and remain visible. (C) During treatment of patient 3, the endogenous FLC-kappa M-protein comigrates with the t-mAbs and can't be distinguished as a separate monoclonal band (left panel). Only the double hydrashift (right) allows correct IFE-interpretation (IFE-negative) of the M-protein. The timepoint of serum sampling is indicated for each patient in the left panel. The M-protein, daratumumab and nivolumab are highlighted in each panel with respectively green, blue and red arrow-heads.

M-protein monitoring is most difficult in patients that respond well to the therapy and have an IgG-kappa or FLC-kappa M-protein that co-migrates with the t-mAb(s). In this situation the endogenous M-protein band cannot be distinguished from $\mathrm{t}-\mathrm{mAb}$ bands, which makes valid clinical response calls that meet the IMWG criteria impossible. Here, we show that additional reflex testing with a double hydrashift assay allows IFE M-protein interpretation even in the presence of two t-mAbs. Advantage of the double hydrashift assay is that it can be easily performed using analyzers already in place, since it involves only a simple modification of the routine IFE protocol. A limitation of the daratumumab/nivolumab hydrashift assay is that it will not abrogate interference of other $t$-mAbs. Therefore, with the introduction of novel (combinations of) t-mAbs, each time new hydrashift assays must be developed, and 
FDA-approved, using antisera specific for these novel t-mAbs. Currently, the daratumumab Hydrashift assay (Sebia) is the only commercially available FDA-approved IFE-assay to mitigate antibody interference [19].

Another limitation of the hydrashift assay is that it is a qualitative assay. In this manuscript we show proof-ofprinciple that one multiplex MS-MRD assay allows quantitative monitoring of the endogenous M-protein and all the $\mathrm{t}$-mAbs that are dosed simultaneously. The MS-characteristics of t-mAbs that are used for the treatment of MM patients and that have previously been published, can be readily implemented in non-quantitative top-down MS and quantita tive bottom-up MS methods [21, 22]. For quantification of M-protein-specific clonotypic peptides, a two-step approach is needed which affects the turn-around-time of the first measurement. From shotgun MS performed on a baseline sample, clonotypic peptides are first identified by aligning the M-protein primary sequence to the most homologous germline variable region of the IMGT reference directory (www.imgt.org). For all subsequent samples of that same patient, the M-protein clonotypic peptides and t-mAb specific peptides are measured and quantified using targeted MS [33]. Advantage of this bottom-up MS method is that it allows extremely sensitive mAb measurements down to $1 \mathrm{mg} / \mathrm{L}$. Disadvantage of bottom-up MS is that it is currently not yet widely implemented in clinical laboratories. Because of improved anti-myeloma therapy an increased number of MM-patients reach a status of (stringent) CR [15], and more sensitive methods to measure $\mathrm{M}$-protein are needed to be able to monitor these patients.

In conclusion, routine M-protein diagnostics becomes increasingly challenging now that the first myeloma patients are treated with combinations of t-mAbs. In this study we show that both daratumumab and nivolumab interfere with electrophoretic M-protein diagnostics. In addition, it is expected that combined t-mAb treatments may further improve the rates and depth of therapyresponse which requires increased assay-sensitivity to monitor disease activity. To anticipate on possible interference of t-mAbs treatment in M-protein monitoring, it is important that the laboratory specialist is informed on the use of monoclonal antibodies in MM treatment. We here describe two different methods to successfully circumvent $\mathrm{t}$-mAb interference, using double hydrashift assays and MS-MRD assays.

Acknowledgments: We thank Sebia's R\&D team for the design and realization of the double hydrashift experiments. We thank Bristol-Myers Squibb and Janssen Pharmaceuticals for supporting the clinical study (NCT03184194).
Research funding: NvdD received research support from Janssen Pharmaceutical, Amgen, Celgene, Novartis, Cellectis, and Bristol-Myers Squibb. JFMJ received a Dutch Cancer Society Grant (\#10817) and research support from Sebia. The other authors have nothing to disclose.

Author contributions: All authors have accepted responsibility for the entire content of this manuscript and approved its submission.

Competing interests: NvdD received research support from Janssen Pharmaceutical, Amgen, Celgene, Novartis, Cellectis, and Bristol-Myers Squibb; Advisory boards for Janssen Pharmaceuticals, Amgen, Celgene, Bristol-Myers Squibb, Novartis, Roche, Takeda, GSK, Sanofi, Bayer and Servier. JFMJ received a Dutch Cancer Society Grant (\#10817) and research support from Sebia. The other authors state no conflict of interest.

Informed consent: Informed consent was obtained from all individuals included in this study.

Ethical approval: The study site Ethics Committee (METc VUmc, study registration number 2017.159/ NL60544.029.17) approved the protocols, which were conducted according to the principles of the Declaration of Helsinki, the International Conference on Harmonization, and the Guidelines for Good Clinical Practice.

\section{References}

1. Cuesta-Mateos C, Alcaraz-Serna A, Somovilla-Crespo B, MunozCalleja C. Monoclonal antibody therapies for hematological malignancies: not just lineage-specific targets. Front Immunol 2017;8:1936.

2. Touzeau C, Moreau P, Dumontet C. Monoclonal antibody therapy in multiple myeloma. Leukemia 2017;31:1039-47.

3. Mateos MV, Dimopoulos MA, Cavo M, Suzuki K, Jakubowiak A, Knop $\mathrm{S}$, et al. Daratumumab plus bortezomib, melphalan, and prednisone for untreated myeloma. N Engl J Med 2018;378:518-28.

4. Facon T, Kumar S, Plesner T, Orlowski RZ, Moreau P, Bahlis N, et al. Daratumumab plus lenalidomide and dexamethasone for untreated myeloma. N Engl J Med 2019;380:2104-15.

5. Moreau P, Attal M, Hulin C, Arnulf B, Belhadj K, Benboubker L, et al. Bortezomib, thalidomide, and dexamethasone with or without daratumumab before and after autologous stem-cell transplantation for newly diagnosed multiple myeloma (CASSIOPEIA): a randomised, open-label, phase 3 study. Lancet 2019;394:29-38.

6. Lokhorst HM, Plesner T, Laubach JP, Nahi H, Gimsing P, Hansson M, et al. Targeting CD38 with daratumumab monotherapy in multiple myeloma. N Engl J Med 2015;373:1207-19.

7. Lonial S, Weiss BM, Usmani SZ, Singhal S, Chari A, Bahlis NJ, et al. Daratumumab monotherapy in patients with treatment-refractory multiple myeloma (SIRIUS): an open-label, randomised, phase 2 trial. Lancet 2016;387:1551-60.

8. van de Donk N, Richardson PG, Malavasi F. CD38 antibodies in multiple myeloma: back to the future. Blood 2018;131:13-29. 
9. Lesokhin AM, Ansell SM, Armand P, Scott EC, Halwani A, Gutierrez $M$, et al. Nivolumab in patients with relapsed or refractory hematologic malignancy: preliminary results of a phase lb study. J Clin Oncol 2016;34:2698-704.

10. Verkleij CPM, Jhatakia A, Broekmans MEC, Frerichs KA, Zweegman S, Mutis T, et al. Preclinical rationale for targeting the PD-1/PD-L1 axis in combination with a CD38 antibody in multiple myeloma and other CD38-positive malignancies. Cancers 2020;12:3713.

11. McCudden CR, Jacobs JFM, Keren D, Caillon H, Dejoie T, Andersen K. Recognition and management of common, rare, and novel serum protein electrophoresis and immunofixation interferences. Clin Biochem 2018;51:72-9.

12. van de Donk NW, Otten HG, El Haddad O, Axel A, Sasser AK, Croockewit $S$, et al. Interference of daratumumab in monitoring multiple myeloma patients using serum immunofixation electrophoresis can be abrogated using the daratumumab IFE reflex assay (DIRA). Clin Chem Lab Med 2016;54:1105-9.

13. Durie BG, Miguel JF, Blade J, Rajkumar SV. Clarification of the definition of complete response in multiple myeloma. Leukemia 2015;29:2416-7.

14. Murata K, McCash SI, Carroll B, Lesokhin AM, Hassoun H, Lendvai N, et al. Treatment of multiple myeloma with monoclonal antibodies and the dilemma of false positive M-spikes in peripheral blood. Clin Biochem 2018;51:66-71.

15. Kumar S, Paiva B, Anderson KC, Durie B, Landgren O, Moreau P, et al. International Myeloma Working Group consensus criteria for response and minimal residual disease assessment in multiple myeloma. Lancet Oncol 2016;17:e328-46.

16. van de Velde HJ, Liu X, Chen G, Cakana A, Deraedt W, Bayssas M. Complete response correlates with long-term survival and progression-free survival in high-dose therapy in multiple myeloma. Haematologica 2007;92:1399-406.

17. McCudden C, Axel AE, Slaets D, Dejoie T, Clemens PL, Frans S, et al. Monitoring multiple myeloma patients treated with daratumumab: teasing out monoclonal antibody interference. Clin Chem Lab Med 2016;54:1095-104.

18. Thoren KL, Pianko MJ, Maakaroun Y, Landgren CO, Ramanathan LV. Distinguishing drug from disease by use of the hydrashift 2/4 daratumumab assay. J Appl Lab Med 2019;3:857-63.

19. Shah N, Aiello J, Avigan DE, Berdeja JG, Borrello IM, Chari A, et al. The Society for Immunotherapy of Cancer consensus statement on immunotherapy for the treatment of multiple myeloma. J Immunother Canc 2020;8:e000734.

20. Zajec M, Langerhorst P, VanDuijn MM, Gloerich J, Russcher H, van Gool AJ, et al. Mass spectrometry for identification, monitoring, and minimal residual disease detection of M-proteins. Clin Chem 2020;66:421-33.

21. Zajec M, Jacobs JFM, Groenen P, de Kat Angelino CM, Stingl C, Luider TM, et al. Development of a targeted mass-spectrometry serum assay to quantify M-protein in the presence of therapeutic monoclonal antibodies. J Proteome Res 2018;17:1326-33.
22. Mills JR, Kohlhagen MC, Willrich MAV, Kourelis T, Dispenzieri A, Murray DL. A universal solution for eliminating false positives in myeloma due to therapeutic monoclonal antibody interference. Blood 2018;132:670-2.

23. Kohlhagen MC, Mills JR, Willrich MAV, Dasari S, Dispenzieri A, Murray DL. Clearing drug interferences in myeloma treatment using mass spectrometry. Clin Biochem 2021 Mar 7. https://doi. org/10.1016/j.clinbiochem.2021.02.011 [Epub ahead of print].

24. Caillon H, Irimia A, Simon JS, Axel A, Sasser AK, Scullion MJ, et al. Overcoming the interference of daratumumab with immunofixation electrophoresis (IFE) using an industrydeveloped dira test: hydrashift 2/4 daratumumab. Blood 2016; 128:2063.

25. Lefranc MP. IMGT, the international ImMunoGeneTics information system. Cold Spring Harb Protoc 2011;2011:595-603.

26. Langerhorst $P$, Brinkman AB, VanDuijn MM, Wessels $H$, Groenen $P$, Joosten I, et al. Clonotypic features of rearranged immunoglobulin genes yield personalized biomarkers for minimal residual disease monitoring in multiple myeloma. Clin Chem 2021;67:867-75.

27. Ye J, Ma N, Madden TL, Ostell JM. IgBLAST: an immunoglobulin variable domain sequence analysis tool. Nucleic Acids Res 2013; 41:W34-40.

28. Zajec M, Jacobs JFM, de Kat Angelino CM, Dekker LJM, Stingl C, Luider TM, et al. Integrating serum protein electrophoresis with mass spectrometry, a new workflow for M-protein detection and quantification. J Proteome Res 2020;19:2845-53.

29. Turner KA, Frinack JL, Ettore MW, Tate JR, Graziani MS, Jacobs JFM, et al. An international multi-center serum protein electrophoresis accuracy and $M$-protein isotyping study. Part I: factors impacting limit of quantitation of serum protein electrophoresis. Clin Chem Lab Med 2020;58:533-46.

30. Jacobs JFM, Turner KA, Graziani MS, Frinack JL, Ettore MW, Tate JR, et al. An international multi-center serum protein electrophoresis accuracy and M-protein isotyping study. Part II: limit of detection and follow-up of patients with small M-proteins. Clin Chem Lab Med 2020;58:547-59.

31. Durie BG, Harousseau JL, Miguel JS, Blade J, Barlogie B, Anderson K, et al. International uniform response criteria for multiple myeloma. Leukemia 2006;20:1467-73.

32. Ludwig H, Miguel JS, Dimopoulos MA, Palumbo A, Garcia Sanz R, Powles R, et al. International Myeloma Working Group recommendations for global myeloma care. Leukemia 2014;28: 981-92.

33. Zajec M, Frerichs KA, Vanduijn MM, Nijhof IS, Stege CAM, AvetLoiseau $\mathrm{H}$, et al. Cerebrospinal fluid penetrance of daratumumab in leptomeningeal multiple myeloma. Hemasphere 2020;4:e413.

Supplementary Material: The online version of this article offers supplementary material (https://doi.org/10.1515/cclm-2021-0399). 